Les espaces publics au prisme de l'art à Johannesburg (Afrique du Sud) quand la ville fait œuvre d'art et l'art œuvre de ville

\title{
Pauline Guinard
}

\section{(2) OpenEdition}

Journals

Édition électronique

URL : http://journals.openedition.org/cdg/1100

DOI : $10.4000 /$ cdg. 1100

ISSN : 2107-7266

Éditeur

UMR 245 - CESSMA

\section{Référence électronique}

Pauline Guinard, « Les espaces publics au prisme de l'art à Johannesburg (Afrique du Sud) quand la ville fait œuvre d'art et l'art œuvre de ville », Carnets de géographes [En ligne], 5 | 2013, mis en ligne le 01 janvier 2013, consulté le 23 septembre 2020. URL : http://journals.openedition.org/cdg/1100 ; DOI : https://doi.org/10.4000/cdg.1100

\section{(c) $(1)$}

La revue Carnets de géographes est mise à disposition selon les termes de la Licence Creative Commons Attribution - Pas d'Utilisation Commerciale - Pas de Modification 4.0 International. 


\section{LES ESPACES PUBLICS AU PRISME DE L'ART À JOHANNESBURG (AFRIQUE DU SUD) QUAND LA VILLE FAIT CEUVRE D'ART ET L'ART CEUVRE DE VILLE}

\section{PAULINE GUINARD}

Cette thèse porte sur les espaces publics à Johannesburg, capitale économique de l'Afrique du Sud. Dans le contexte post-apartheid, I'utilisation de la notion d'espaces publics pose problème : d'une part, du fait des ségrégations passées qui ont eu tendance à faire de ces espaces des lieux de séparation et de mise à distance des différents publics; et d'autre part, du fait des taux de violence élevés et du fort sentiment d'insécurité, qui tendent à encourager la sécurisation et la privatisation de ces espaces. Faut-il alors en conclure qu'il n'y a tout simplement pas d'espaces publics à Johannesburg, ce qui remettrait d'ailleurs en cause la capacité même de Johannesburg à faire ville?

Cette conclusion, sans doute trop hâtive, peut être nuancée si l'on adopte, comme nous nous proposons de le faire, une lecture dynamique et en trois dimensions des espaces publics. En distinguant :

- les espaces juridiquement publics, c'est-à-dire les espaces relevant de la propriété publique,

- les espaces socialement publics, au sens d'espaces de côtoiement et de possible rencontre d'individus dans leur diversité,

- et les espaces politiquement publics, compris comme espaces de libre expression de tout un chacun,

il est en effet possible de mettre en évidence que le sentiment - particulièrement sensible pour un européen - d'un déficit d'espaces publics à Johannesburg s'explique non par une absence totale de ces espaces dans toutes leurs dimensions, mais par l'absence d'une des dimensions de leur publicité (au sens de caractère public), voire par un déphasage de ces trois dimensions. Les espaces juridiquement publics ne s'identifieraient pas systématiquement aux espaces socialement publics, lesquels, à leur tour, ne correspondraient pas spontanément aux espaces politiquement publics, produisant un déphasage tel qu'il parviendrait à anéantir la perception même de l'existence des espaces publics en général.

C'est dans ce contexte que nous entendons comprendre les espaces publics " au prisme de l'art ". En effet, l'art qui se déploie dans les espaces publics se rapporte à toutes leurs dimensions, que ce soit : sur le plan juridique, en tant qu'installé dans les espaces 
appartenant à la puissance publique; sur le plan social, en entendant favoriser la diversification des publics présents dans ces espaces et leur interaction; ou encore sur le plan politique, en promouvant l'expression de tous, y compris des personnes généralement sous-représentées. Dans cette perspective, l'art, en agissant potentiellement sur les espaces publics aussi bien juridiques que sociaux et politiques, est envisagé comme une clef de lecture permettant de rendre visibles et de distinguer les différentes dimensions qui composent ces espaces. De surcroît, en agissant sur les différentes dimensions des espaces publics, l'art pourrait également favoriser un mouvement de " re-phasage » de ces différentes dimensions qui paraissent, de fait, presque inconciliables à Johannesburg. L'utilisation de l'art comme d'un prisme ouvre une double perspective, en ce qu'elle permet:

- d'un côté, de mieux saisir ce qu'est et peut être un espace public à Johannesburg, en mettant à jour les différentes dimensions de leur publicité ;

- et de l'autre, de participer à la (re)construction de leur caractère public dramatiquement affaibli par les précédents régimes politiques, en renforçant l'une de ses dimensions éventuellement défaillante ou en faisant coïncider, le cas échéant, toutes les dimensions à la fois.

Selon une approche dynamique, qui appréhende les espaces publics comme des processus et non comme des objets définis une fois pour toutes, l'enjeu de cette recherche est de mettre en lumière et de comprendre, par l'art, les processus actuels de construction ou de déconstruction du caractère public des espaces johannesburgeois, aussi bien dans leur dimension juridique que sociale et politique.

Adoptant une démarche qualitative, notre étude se base à la fois sur des observations de terrain et des entretiens conduits auprès des producteurs mais aussi des récepteurs de cet art qui a lieu dans les espaces publics johannesburgeois. Six espaces ont plus particulièrement été choisis pour mener nos enquêtes, et ceci afin d'envisager à la fois différents contextes urbains (centre-ville, banlieues aisées et townships - anciens espaces de relégation des populations de couleur) et différentes formes d'art (permanent et temporaire, hérité des périodes antérieures ou récemment créé, financé par des acteurs publics ou privés, etc.). Une telle approche n'est toutefois pas sans poser des difficultés d'ordre méthodologique, non seulement parce qu'elle implique de trouver des procédés susceptibles de saisir l'impact de ces arts sur la publicité des espaces, et notamment sur les pratiques et représentations de leurs usagers, mais aussi parce qu'elle suppose de fréquenter ces espaces réputés - et ce particulièrement pour une jeune femme blanche dangereux.

En dépit mais aussi du fait de ces contraintes, cette thèse permet de mettre à jour trois grandes tendances quant au rôle de l'art dans les espaces publics et, avec eux, dans la ville de Johannesburg (voir photographies ci-dessous), à savoir :

1- un art qui participe à la mise en normes de la ville, celles d'une ville qui se veut africaine et globale ;

2- un art qui tend à rendre normal le passé conflictuel de la ville, en le commémorant pour mieux le mettre à distance et le mettre en vente ; 
3- un art qui prétend au contraire résister à cette double normalisation de la ville par l'art, en proposant une autre conception des espaces publics, plus fluide, mouvante et moins empreinte de peurs.

\title{
L'art à Johannesburg, entre normalisation des espaces publics et résistance à la normalisation
}

1- L'Eland à Braamfontein (centre-ville), symbole d'une Johannesburg africaine et globale ?

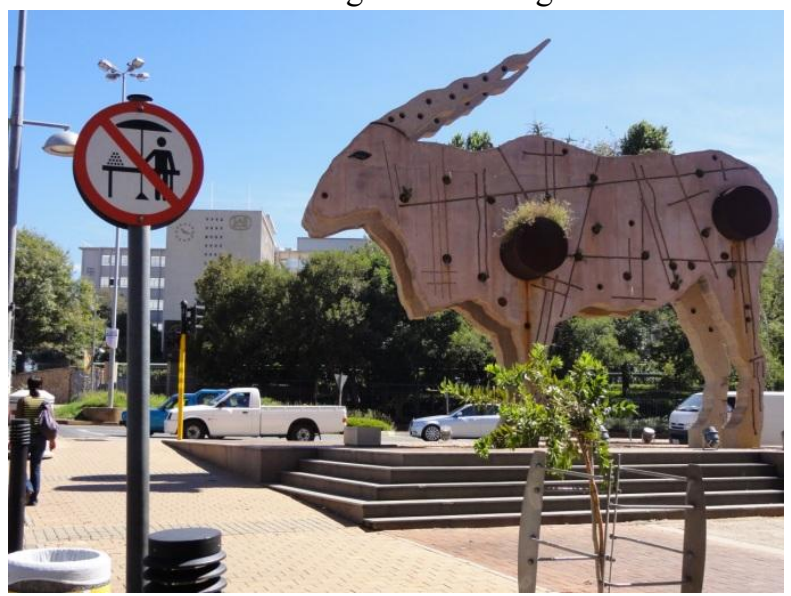

3- Le spectacle des Grandes Marionnettes à Joubert Park (centre-ville) : de l'art pour dépasser ses peurs ?

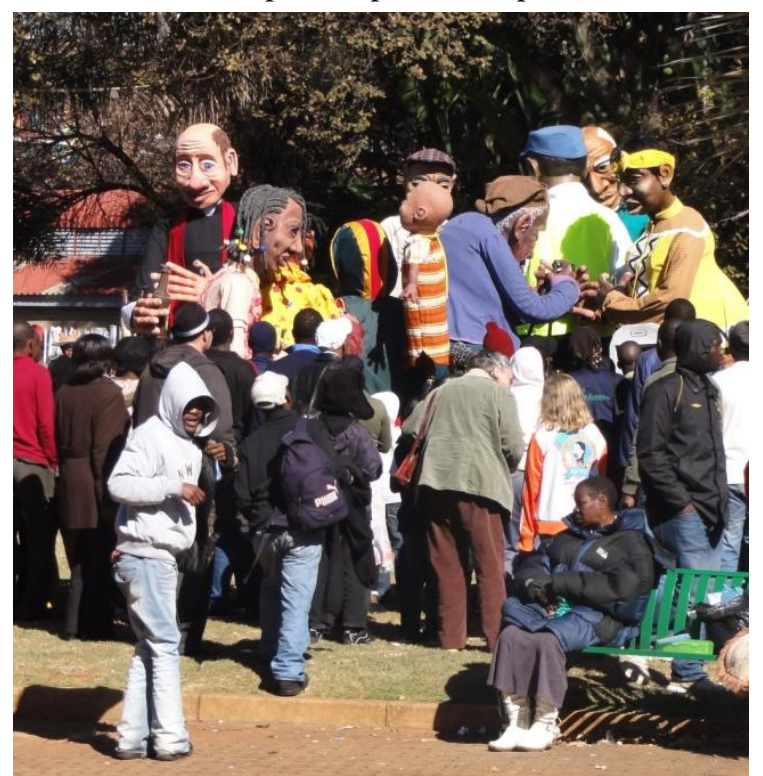

(C) Pauline Guinard, 2011
2- Commémorer les émeutes de 1976 à Orlando West (Soweto) pour mieux mettre le township en tourisme

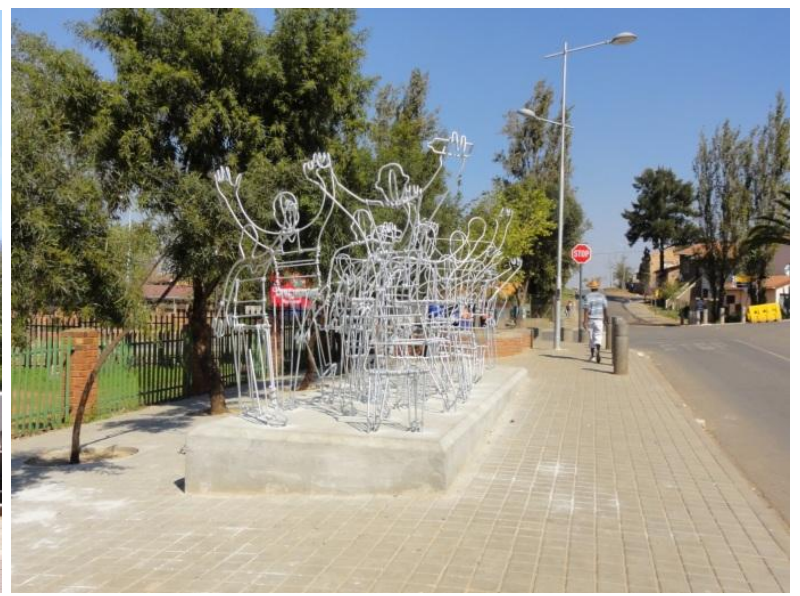

\author{
(1)
}




\section{Discipline}

Géographie

\section{Directeur}

Philippe Gervais-Lambony

\section{Université}

Université Paris Ouest Nanterre la Défense

Membres du jury de thèse, soutenue le 28 novembre 2012

- Marie-Hélène Bacqué, Professeure d'urbanisme, Université Paris Ouest Nanterre la Défense Présidente

- Guy Di Méo, Professeur de géographie, Université Michel de Montaigne, Bordeaux 3

- Philippe Gervais-Lambony, Professeur de géographie, Université Paris Ouest Nanterre la Défense Directeur

- Maria Gravari-Barbas, Professeure de géographie, Université Paris 1 Panthéon Sorbonne Rapporteure

- Myriam Houssay-Holzschuch, Professeure de géographie, Université Joseph Fourier, Grenoble Rapporteure

- Cynthia Kros, Directrice adjointe de l'Ecole d'Art de l'Université du Witwatersrand, Johannesburg

Situation professionnelle actuelle

Agrégée Préparatrice à l'Ecole normale supérieure de Paris

\section{Courriel de l'auteur}

pauline.guinard@gmail.com 